\title{
Una aproximación a la microeconomía no neoclásica de Hicks
}

An approach to microeconomics not neoclassical of Hicks

\section{Resumen}

Para Hicks el reconocimiento microeconómico de los problemas económicos pasa por considerar al menos dos sectores. Así cuando se trata de los precios, el sistema económico se divide en dos sectores: una de precios fijos y otro de precios flexibles, el sector de precios fijos se ajusta mediante las cantidades y tiende a no variar cuando varía la demanda y el sector de los precios flexibles, se corresponde con los mercados de competencia; en el caso del efecto multiplicador de la inversión, hay que dividir los recursos de las empresas en factores de flujo y factores de reserva. Si la demanda se expande, el efecto multiplicador puede reducir o tardar en hacer su efecto, si las empresas tienen muchas reservas de mercancías en inventarios, con las cuales se hace frente a la expansión de la demanda; lo mismo pasa cuando Hicks investiga las repercusiones de los costos salariales en las variaciones de los precios. Para hacerlo, divide el mercado laboral en dos sectores: uno, en el que se tiene una relación laboral estable de largo plazo y está regida por un acuerdo o convenio mutuo entre los trabajadores y los contratantes y otro, donde la relación laboral es efimera y no hay seguridad en el trabajo.

Palabras clave:

- Microeconomía

- Neoclásicos

- Estructura de mercado y formación de precios
Abstract

To Hicks the microeconomic recognition of the economic problems happens because it's considered at least two sectors. So when it comes to prices, the economic system is divided into two sectors: one of fixed prices and another of flexible prices, the fixedprices sector is adjusted by the amounts and tends not to vary when the demand varies and the flexible prices sector, corresponds to competitive markets, in the case of the multiplier effect of investment, it is necessarily to divide the resources of companies in flow factors and factors of booking. If demand expands, the multiplier effect could reduce or take long to make its effect, if companies have many stocks of goods in inventories, which with its faced the expanding demand, the same happens when Hicks investigates the impact wage costs in the price variations. To do this, he divides the labor market into two sectors: one, in which exists a stable employment relationship of long term and is directed by an agreement or mutual agreement between employees and contractors and another, where the employment relationship is ephemeral and there is not security in the job.

\section{Keywords: \\ - Microeconomics \\ - Neoclassical \\ - Market Structure and Pricing}

JEL: B21, E13, D4

\section{Introducción}

Si bien John Richard Hicks es reconocido por su libro Valor y Capital y por sus aportaciones a la síntesis neoclásica en el ámbito de la macroeconomía, no dejan de ser menos meritorias sus indicaciones de cómo construir una teoría microeconómica que siga por caminos diferentes a los trillados por la teoría convencional. En este aspecto, las elaboraciones de Hicks no han sido tan 
Economía Informa núm. 383 noviembre - diciembre • 2013 | " " "

renombradas porque le ha ocurrido como a quienes "traicionan" su afiliación a una doctrina: son rechazados por sus seguidores y no son aceptados por sus adversarios. Sin embargo, dentro de la corriente teórica del poskeynesianismo, habría que hacerle un amplio espacio a lo que son algunas de las contribuciones más relevantes de Hicks en el campo de la teoría microeconómica.

Vale la pena citar una frase de Hicks en una entrevista que la hicieron en Oxford, Inglaterra, en junio de 1977. Ante una larga intervención de su interlocutor sobre su libro La crisis de la economía keynsiana, Hicks responde: "Debería comenzar diciendo que voy a tener que cambiarme de nombre. Valor y Capital (1939) fue escrito por un economista neoclásico llamado J. R. Hicks, que ya murió (subrayado nuestro), mientras, como lo he insinuado en otra parte, los libros Capital y tiempo (1973) y Una teoría de la historia económica (1969) fueron escritos por John Hicks, un economista que no es neoclásico y que le falta al respeto a su tío con frecuencia." (Pizano, 1980: 56-57). Así, podemos reconocer a un Hicks no neoclásico a partir del año de 1965 cuando publica Capital y crecimiento en el siglo pasado. Este es el economista que nos interesa retomar para subrayar sus aportes a la teoría microeconómica no neoclásica.

El ensayo ha sido dividido en tres partes. En la primera se revisan algunas ideas del Hicks neoclásico que ya apuntaban hacia las limitaciones de la teoría económica de la competencia perfecta. En la segunda se presentan los puntos clave de las contribuciones de Hicks: la matriz de clasificación de los mercados. En la parte final se hacen algunas aproximaciones a las implicaciones macroeconómicas de los postulados micro no-neoclásicos de Hicks, haciendo énfasis en el funcionamiento de los mercados en condiciones de globalización de la economía.

Vale la pena, antes de incorporarnos el pensamiento de Hicks de la última etapa de su vida, aclarar que, aunque ya se insinuó, en este ensayo no se van a revisar sus planteamientos neoclásicos, sino todo lo contrario y quien espere reconocer en estas líneas al Hicks convencional va a sufrir una fuerte decepción.

\section{Las limitaciones de la teoría neoclásica según Hicks}

Para muchos economistas, la publicación de Valor y Capital significó la refundación de la teoría neoclásica, tanto en su parte microeconómica, como en su parte macroeconómica. Si bien el libro de Marshall sobre principios de economía, contenía las bases de la teoría neoclásica, había algunas cosas que 
estaban insuficientemente desarrolladas y otras que ameritaban un tratamiento más amplio. Hicks, se propuso llenar esos vacíos y actualizar la teoría.

En el capítulo VI del libro Valor y Capital, al hacer la exposición sobre el equilibrio de la empresa dentro del equilibrio general, Hicks señala que un requisito indispensable para que el modelo teórico tenga aceptación es mantener el supuesto de competencia perfecta. Esto implica, sobre todo, la inexistencia de controles sobre el mercado, es decir, que todos los agentes y sobre todo las empresas son tomadores de precios y de manera individual no tienen ningún poder de mercado para influir sobre los precios. "Hasta aquí todo va bien; pero habremos de reconocer que el abandono general del supuesto de la competencia perfecta, la aceptación universal del supuesto de que existe monopolio, ha de tener consecuencias muy destructoras para la teoría económica." (Hicks, 1976: 93). Entonces, se entiende que sólo se cumplen los requisitos de funcionamiento eficiente y óptimo de los agentes económicos, si se supone que todos los mercados actúan en condiciones de competencia perfecta. "A mi modo de ver sólo se puede salvar algo del naufragio -y recuérdese que el desastre que amenaza abarca la mayor parte de la teoría del equilibrio general- si podemos suponer que los mercados que se ofrecen a la mayor parte de las empresas de que nos ocuparemos no se apartan mucho de aquellos en que rige la competencia perfecta." (Hicks, 1976: 54). El naufragio de la teoría económica se presenta cuando en los mercados del mundo real aparecen empresas con capacidades monopólicas. El grado de monopolio con que se presentan las condiciones de producción en las manufacturas y en los servicios, hacen necesaria la revisión de la teoría convencional referente a la formación y variación de precios y al comportamiento de las estructuras de producción.

Una de las características del método de análisis de Hicks, es la de relacionar los procesos reales de la economía con abstracciones teóricas, o mejor dicho al de observar los fenómenos reales y vincularlos a las elaboraciones teóricas, Por ello estaba preocupado, en la década de los treinta del siglo pasado, con la aparición masiva de empresas con poder monopólico y sus efectos destructores sobre el modelo teórico basado en la competencia perfecta. Para su óptica, estas transformaciones de las estructuras económicas de los países, estaban ya anunciando que la teoría prevaleciente tenía poco o nada que decir sobre la marcha de la economía en el mundo real. Con el desarrollo de las estructuras monopólicas en todos los ámbitos de la economía, Hicks reconoce que los instrumentos teóricos proporcionados por los modelos de competencia perfecta, son no sólo insuficientes, sino inadecuados para estudiar la realidad económica. Y este es un punto clave en el pensamiento de Hicks, porque en 
Economía Informa núm. 383 noviembre - diciembre • 2013 | " " "

la actualidad se sigue enseñando la teoría de la competencia perfecta porque es el más simple y más fácil de comprender, pero no se considera que su explicación del mundo real es nula y que las estructuras monopólicas en los mercados parten de bases diferentes y llegan a conclusiones disímiles. Esto es lo que ya anunciaba Hicks en 1939, cuando indicaba que la presencia de monopolios en los mercados hace naufragar la teoría económica del equilibrio parcial y general y que sólo suponiendo que las diferencias entre los precios y los costos marginales "no son muy grandes ni muy variables" se pueden asimilar las leyes de un sistema económico que contenga un volumen crecido de elementos monopólicos a un sistema económico que actúa bajo condiciones de competencia perfecta. "Parece que cuando menos vale la pena intentar esa escapatoria. Pero debemos darnos cuenta de que estamos dando un paso peligroso y quizá limitando en gran magnitud la amplitud de los problemas que se puedan encajar en nuestro análisis subsiguiente.” (Hicks, 1976: 94). La mejor manera de seguir adelante es suponer que en la realidad no existen los monopolios o cualquier grado de monopolio y así aceptar que es la competencia perfecta la que rige en las economías reales. Hicks concluye esta advertencia diciendo "A pesar de ello, dudo que sea posible hacer un análisis útil de la mayor parte de los problemas que habremos de excluir por esta razón si empleamos los métodos de la teoría económica" (Hicks, 1976:94). Es decir, si se siguen empleando los modelos de competencia perfecta, los análisis que se hagan serán inútiles. Con esto, nos estaba anunciando ya que la teoría neoclásica convencional estaba a punto de ser superada por la evolución de la realidad económica y se necesitaba otra clase de método en la teoría económica que diera cuenta de los fenómenos nuevos: una economía dominada por los monopolios en diferentes grados o por empresas con poder de mercado.

Pero, la competencia perfecta no es el único supuesto que limita los hallazgos del libro que Hicks escribió para apuntalar la teoría neoclásica. La hipótesis sobre los tipos de bienes que se comercian, también limitan el análisis. En el mercado de bienes y servicios, el análisis convencional, está referido a los bienes y servicios que tienen precios totalmente flexibles. Es decir, el ajuste del mercado, cuando no hay coincidencia entre la oferta y la demanda, se logra a través del movimiento inmediato del precio. Esto puede ser válido solamente para aquellos tipos de bienes que no pueden ser acumulados, o guardados en inventarios. Así, la hipótesis de Valor y Capital es que los bienes que se comercian son bienes perecederos, servicios personales y bienes especulativos. Los dos primeros no pueden ser guardados en existencias para periodos subsiguientes y los bienes especulativos se acumulan hoy con el único fin de 
vender a un mayor precio en el futuro. En ambos casos los ajustes del mercado se dan a través del cambio en los precios. A ese campo de estudio se restringe el análisis del mercado en el libro citado. Por ello, es bastante limitado, pues es bien sabido que la mayoría de los bienes manufacturados no se mueven en mercados especulativos y no son bienes perecederos.

Todo este campo queda fuera del análisis de Hicks en Valor y Capital. Y es el grupo de bienes donde la innovación y el desarrollo tecnológico y por tanto la dinámica económica de la sociedad son más importantes. Como dice el propio Hicks "El error del análisis de Valor y Capital es su tratamiento de un tipo de mercado excepcional como si fuese común" (Hicks, 1989: 212). El mercado de precios flexibles es la excepción y no puede ser tomado como la generalidad en el mundo real.

\section{Las contribuciones de Hicks a la teoría microeconómica no-neoclásica. El método en Hicks}

Para Hicks, el método consiste en el modelo teórico y su esfuerzo por aclarar el método estriba en construir teoría económica que explique la realidad en su contexto histórico. Para lograrlo trabaja desde un punto de vista estático hacia una visión dinámica de la economía. Para él, los pasos más importantes en el desarrollo teórico se han dado en la construcción de modelos dinámicos en donde sus aportaciones han sido significativas. "Uno de los mayores cambios que ha experimentado la teoría económica en los últimos 30 años es la transformación de la dinámica económica, de una aspiración reverente, en un respetable cuerpo de principios." (Hicks, 1989: 206). ¿En qué consiste ese respetable cuerpo de principios?

En el capítulo 18 de Métodos del análisis dinámico, Hicks sintetiza lo que desde mi punto de vista es su concepción del método adecuado para definir los diferentes mercados que pueden encontrarse en un sistema económico, por eso habla de que se aclaró su método cuando logró una clasificación.

La clasificación de los mercados la hace con base en una matriz de 2 por 2 como la siguiente:

\begin{tabular}{|c|c|c|}
\hline & Modelos P & Modelos Q \\
\hline Modelos ex ante-ex post & $1 \mathrm{a}$ & $1 \mathrm{~b}$ \\
\hline Modelos de stocks y flujos & $2 \mathrm{a}$ & $2 \mathrm{~b}$ \\
\hline
\end{tabular}


Economía Informa núm. 383 noviembre - diciembre • 2013 ㅁ

Donde los modelos $\mathrm{P}$ son los que se refieren a precios flexibles; los modelos Q a precios fijos; los modelos ex ante-ex post incorporan el tiempo y los modelos de stocks-flujos se basan en la contabilidad comercial (Hicks, 1989).

Con esos cuatro modelos se pueden clasificar los mercados en cuatro tipos, además de los híbridos. El primer mercado, con precios flexibles a través del tiempo (1a) es el que se refiere a los modelos que se describen en su libro Valor y Capital. En este libro la característica central no es sólo que se haya restringido a un mercado de competencia perfecta, sino que el tipo de bienes tiene que ser estudiado a través del ajuste en los precios. Lo que son los precios y lo que se espera que sean, determina la dinámica del mercado: "los precios y no las cantidades tendían a resultar diferentes de los esperados. Las demandas... serían siempre iguales a las ofertas...en términos de las cantidades; las diferencias aleatorias entre la expectativa y la realización se imputaban a los precios." (Hicks, 1989. 211).

Con el supuesto de los precios absolutamente flexibles hacia arriba y hacia abajo, el ajuste de las diferencias entre lo esperado y lo ocurrido, se da a través de los precios y no de las cantidades (la cantidad ofrecida siempre es igual a la cantidad demandada, por medio de los cambios en los precios). El tipo de bienes que se ajusta a este tipo de mercado tiene que ver con la no durabilidad de los mismos y con la inmediatez de los mercados. Entre éstos tenemos a 1. Los bienes perecederos sin posibilidad de acumular existencias por mucho tiempo. Y, 2. Los servicios que no se pueden llevar de un periodo a otro. En ambos casos los precios están determinados por una oferta dada y los cambios que ocurren en la demanda. Entre los bienes perecederos, se pueden nombrar los animales extraídos del mar y los bienes agrícolas de poco tiempo de duración en buen estado y por tanto rápidamente perecederos ( $\sin$ tomar en cuenta las nuevas capacidades tecnológicas para conservar este tipo de bienes en buen estado). Y entre los servicios pueden nombrarse los personales que se negocian cotidianamente y los servicios que pueden bajar o subir de precio según sean la oferta y la demanda de los mismos, por ejemplo, ciertos tipos de servicios profesionales. que nos dan las subastas donde los bienes son irreproducibles o son bienes perecederos. Son ex-ante, cuando salen al mercado con un precio estipulado y son ex-post, cuando se fija el precio después de las negociaciones entre los el vendedor y el comprador.

Los mercados particulares en donde se encuentran los bienes que ajustan sus desequilibrios por medio de cambios en los precios y que a su vez tienen en cuenta los stocks acumulados, 2a en nuestra clasificación, se denominan mercados especulativos. En este tipo de mercado las existencias juegan un papel clave, el grado 
de que el precio se determina por las condiciones en que la demanda de inventarios se iguales a la oferta de los mismos. En este tipo de mercados encontramos normalmente a los comerciantes al mayoreo y a veces también los detallistas. En este tipo de empresas siempre existen acervos acumulados, los cuales cambian su nivel dependiendo de los movimientos de los consumidores y de los productores. Si crece la demanda los stocks disminuyen y si crece la producción los stocks aumentan, pero es el comerciante el que se da cuenta de los niveles de sus acervos, por lo que finalmente serán los niveles de existencias los que determinarán los cambios en los precios. "Por lo tanto, es cierto que en cada momento dado se determina el precio por la demanda y la oferta de existencias." (Hicks, 1989. 213). El ejemplo más claro de este tipo de mercado es el de los granos a nivel internacional. Los precios del trigo, del maíz, del arroz, cambian cuando los niveles de inventarios a nivel internacional, los grandes comerciantes de granos, estiman que los stocks han bajado de su nivel "normal" y además tienen expectativas de que así sigan en al futuro.

En el caso de los mercados de precios fijos (1b), se está suponiendo que los flujos de oferta y de demanda no afectan los precios o que su efecto sobre el movimiento de los precios es apenas significativo. Las políticas de precios de las empresas manufactureras, que tienen como política inventarios cero, están determinadas por los objetivos generales de la empresa. No importa que los flujos de oferta y de demanda estén en desequilibrio, si al precio fijado la demanda es menor a la oferta, la empresa acumulará inventarios no deseados, pero no por eso, necesariamente, va a modificar su precio. Si la acumulación de inventarios no deseados persiste, la empresa modificará sus políticas, no sólo y exclusivamente la de precios, para ajustarse a la nueva situación. Puede, desde bajar sus niveles de producción, hasta aumentar sus gastos de venta (publicidad) para ganar mayores porciones del mercado. En este tipo de mercado los stocks juegan un papel clave para el diseño de las políticas de las empresas. Por ello diremos que los que más requieren las empresas que se mueven en este tipo de mercados, es conseguir, sobre todo, un equilibrio de stocks, en el tiempo. Las empresas manufactureras son el tipo más claro de empresas con precios fijos a lo largo del tiempo. Precios fijos no quiere decir que no puedan cambiar, sino que al enfrentar, a lo largo del tiempo, desequilibrios de oferta y demanda, no necesariamente se van a resolver mediante cambios en los precios.

Por último la celda de la matriz donde se encuentran los mercados de precios fijos y con el manejo de flujos y stocks (2b), es posible afirmar que es el tipo de mercado más común en la actualidad. En ellos los equilibrios de flujos 
Economía Informa núm. 383 noviembre - diciembre • 2013 | " " "

no tienen importancia, pero los flujos sirven para restablecer los desequilibrios de acervos. La mayor parte de los bienes en la actualidad caen en esta categoría. Los bienes manufacturados y los bienes agrícolas y primarios que pueden ser guardados en grandes cámaras de refrigeración para usarlos cuando se requieran. En todos estos casos el equilibrio de inventarios es la base para analizar la marcha de la empresa. De acuerdo con su experiencia cada empresa puede decir qué nivel de inventarios, registrado en su balance, es el mejor entre las opciones que puede manejar según sus anotaciones históricas. "Así pues, el desequilibrio de existencias es el motor, o por lo menos una parte del motor, que impulsa el proceso que estamos analizando" (Hicks, 1989, 219).

\section{Implicaciones macroeconómicas de las teorías microeconómicas de Hicks}

En este apartado se van a tomar en cuenta las relaciones entre los mercados de precios fijos y precios flexibles, así como las condiciones macroeconómicas de un país, cerrado y abierto, y las circunstancias en que los mercados se desenvuelven en la globalización.

En donde Hicks analiza la relación entre los mercados de precios fijos y sus repercusiones en los niveles macroeconómicos, es en el libro titulado "La crisis de la economía Keynesiana". Como él mismo dice, este libro es "una versión revisada de las conferencias que di en Helsinki... del 24 al 26 de abril de 1973" (Hicks, 1976, 7) y contiene importantes aportaciones al análisis macroeconómico desde una perspectiva microeconómica no convencional.

Si se dividen en dos grandes apartados los problemas macroeconómicos, en estabilidad interna o tasas inflacionarias y en nivel de actividad económica y crecimiento, es posible ordenar el pensamiento microeconómico de Hicks para delimitarlos.

En el caso de la estabilidad, o sea la inflación en un sistema económico, es importante saber el peso o el tamaño en cada economía, de los sectores que operan con precio flexibles y los que funcionan bajo el esquema de precios fijos. Cuando el sector de precios fijos cubre la mayor parte del las actividades económicas de un sistema, entonces lo precios no responden a la escasez: "La demanda puede quedar insatisfecha, el registro de pedidos se agrandará y eso es todo." (Hicks, 1976b, 80). La expansión de la demanda no necesariamente va a provocar un aumento de precios y menos aún si las empresas trabajan con capacidades productivas ociosas. En el mismo sentido, si para estas empresas se elevara el precio de las materias primas que utiliza, y esta elevación es tem- 
poral, no tiene por qué aumentar el precio de los bienes terminados, ya que estos son vendidos en mercados de precios fijos "donde los precios se basan en los costos normales y no en los costos del momento" (Hicks, 1976b, 80). Así pues, en un sistema económico donde las empresas de precios fijos tengan dominancia, la expansión de la demanda, ya sea de bienes finales o de materias primas, no necesariamente se traducirá en un aumento generalizado del nivel de precios, es decir, que no necesariamente habrá inflación.

Por el lado de los costos salariales y su influencia sobre los precios, se debe de tomar en cuenta también una división de la economía en dos sectores: aquellos que se manejan con empleo casual o temporal y aquellos sectores de la economía que funcionan con un empleo regular o de largo plazo. Cuando, dentro de un sistema económico, la mayor parte del empleo es regular, eso hace suponer que entre los patrones y los trabajadores hay un acuerdo para mantener los salarios independientemente del nivel de desempleo que exista. Cuando el trabajo es temporal tiende a ser precario y los salarios dependen de la oferta de mano de obra en relación a la demanda. En los sectores de empleo regular, los salarios no se incrementan cuando la mano de obra escasea, ni tampoco se reducen los salarios cuando aumenta la desocupación. Es decir, que en el mercado de trabajo, el salario no se establece por la escasez o abundancia de mano de obra, sino por las negociaciones de largo plazo entre los trabajadores y los empresarios; por lo tanto el salario no va a depender del nivel de empleo sino de las capacidades financieras de las empresas, que están cimentadas en sus ganancias corrientes y esperadas.

Así, la inflación no necesariamente va a depender directamente de los excesos de demanda sobre la oferta cuando en el sistema económico predominan las empresas enclavadas en mercados de precios fijos; ni tampoco, la inflación va a depender de las variaciones en el nivel de ocupación, pues éste no afecta el salario cuando la mayor parte de la mano de obra se ubica en el sector de trabajo regular o de largos periodos.

En lo que respecta al nivel de actividad económica y el crecimiento, la primera idea que se puede exponer de Hicks, es la relacionada con el multiplicador de la inversión y la pregunta clave es ¿cuánto tiempo tardan en sentirse los efectos del multiplicador en una economía que incrementa su inversión? Tomando el ejemplo que Hicks expone, si la inversión se amplía para construcción de vivienda, esto supone que existen en reservas los materiales de construcción (ladrillos) y si no existen, habrá que esperar que se produzcan los materiales adicionales. Pero suponiendo que existan stocks en cantidades suficientes para hacer frente al nuevo nivel de inversión, los productores de 
Economía Informa núm. 383 noviembre - diciembre • 2013 | " " "

materiales no deciden aumentar su producción hasta que vean a qué nuevo nivel se sitúan sus reservas. La reactivación económica depende del nivel de stocks de los materiales esenciales y de las decisiones de quienes producen esos materiales. El producto y el empleo no necesariamente crecen multiplicadamente cuando se aumenta la inversión. De hecho la inversión bruta queda disminuida cuando se le resta la desinversión de los inventarios de materiales. Así, el efecto multiplicador quedará reducido con el uso de materiales para la producción preexistentes en inventarios.

Si no existen reservas suficientes de ladrillos y la economía está abierta al comercio exterior, puede enfrentarse ese cuello de botella mediante importaciones. Entonces el problema se traslada a la balanza de pagos. Su funcionamiento dependerá de las reservas de divisas. Y aquí la expansión puede ser obstaculizada por la crisis de la balanza de pagos. La escasez de medios de pago internacional definirá el límite de la reactivación. Nuevamente son las reservas, en este caso de divisas, las que permitirán que la reactivación sea más o menos amplia.

Relacionado con el comercio exterior, un último tema que se tratará es el que se relaciona con la globalización. En la medida en que las condiciones del mercado internacional se van imponiendo sobre las naciones, es necesario revisar cómo funciona el mercado mundial y cómo este afecta a cada una de las naciones implicadas en el intercambio de bienes y servicios.

La globalización tiene muchos aspectos, pero son dos los que se resaltarán en este escrito: la inversión directa extrafronteras y los flujos de comercio relacionados con ella. ¿Cuál es el precio de una mercancía en el mercado mundial? Sería difícil, si no es que imposible, hallar un precio único para un bien o servicio. ¿Por qué? Porque el comercio internacional está dominado por grandes empresas que actúan en un mercado oligopólico que compite mediante la diferenciación de sus productos. Y por supuesto que en cada país, según las condiciones, el precio será puesto por las empresas que producen el producto. Ni siquiera para el mismo producto, de la misma empresa habrá un precio único en todos los países donde se comercializa ese producto.

La globalización ha traído, como una de sus principales características, la fragmentación de la producción de una empresa en diferentes países. Siguiendo el método de Hicks, de particionar los diferentes tipos de transacciones, entonces se puede observar cómo han evolucionado los mercados en la globalización.

Si la empresa matriz que está situada en los Estados Unidos, tiene varias filiales en varios países y estas filiales producen las partes para el producto 
final que se elabora en los Estados Unidos, entonces se tendrá un comercio intrafirma que hará que el valor de las mercancías trasladadas de un país a otro crezca, es decir, que el comercio internacional crece en valor, pero en realidad el comercio intrafirma no puede considerarse comercio de bienes, pues se realiza dentro de una misma empresa que tiene instalaciones en diferentes países. Antes al contrario, en la medida en que el comercio intrafirma crece (según estimaciones de la OECD ha alcanzado un tercio del comercio internacional), se puede afirmar que el comercio de bienes entre empresas diferentes, situadas en diferentes países, no crece. Crece el envío de partes y componentes dentro de la empresa y esto, en la medida en que no es comercio entre diferentes propietarios, no puede ser considerado comercio de mercancías. Propiamente estos artículos no entran a un mercado de compra y venta, sino que son un traslado intrafirma que se rige por otras reglas, o mejor dicho que se rige por la estrategia de cada empresa en particular y no por las condiciones de los mercados. Aunque es de reconocer que el mercado del bien final tiene influencia sobre esta estrategia de las empresas su intercambio al interior de la empresa, aunque traspase fronteras no deja de ser eso: un movimiento de bienes al interior de la empresa. El mercado, de este tipo de mercancías, no existe en la realidad y por tanto se puede decir que la globalización, y sobre todo la inversión extranjera directa ha traído como consecuencia el aumento del valor del comercio internacional, pero, al mismo tiempo, la reducción del mercado internacional. Lo que se está imponiendo no es un mercado entre naciones, sino un movimiento de bienes al interior de las empresas transnacionales que están efectuando su producción en varias partes del planeta. Por esta misma razón se puede concluir que el comercio intrafirma está creciendo y que ello trae como consecuencia la reducción de la capacidad de los gobiernos para incidir en su comercio exterior.

En la globalización se tiene un mercado tradicional de bienes y de servicios y un mercado o pseudo-mercado, donde lo que se traslada son partes y componentes, dentro de una comercio intrafirma que está determinado por la estrategia de la gran empresa transnacional. Y esto hace que los precios de los bienes se rijan más por esta estrategia que por las fuerzas del mercado. A nivel del sistema mundial no sólo se tiene un mercado internacional formado por empresas oligopólicas, sino además, un mercado intrafirma controlado plenamente por las mismas empresas. La competencia en el mundo se desarrolla a partir de las decisiones de las grandes empresas y por ello suponer que el mercado internacional es más competitivo que el nacional, es una apreciación mal orientada. 
Economía Informa núm. 383 noviembre - diciembre • 2013 | " " "

\section{Conclusiones}

El método empleado por Hicks para ahondar en los linderos de la microeconomía consiste en trabajar con dos sectores en vez de uno solo, como lo supone la macroeconomía. Un solo producto (PIB), con un solo individuo como consumidor (C) y un solo inversor en el ámbito privado (I), así como un solo gobierno y el sector externo tomado como una única vía de intercambio con el exterior, son las variables tradicionales en el análisis macroeconómico. El método microeconómico de Hicks consiste en partir de un sistema económico que tiene más de un sector.

Para Hicks el reconocimiento microeconómico de los problemas económicos pasa por considerar al menos dos sectores. Así cuando se trata de los precios, el sistema económico se divide en dos sectores: una de precios fijos y otro de precios flexibles, el sector de precios fijos se ajusta mediante las cantidades y tiende a no variar cuando varía la demanda y el sector de precios flexibles y ex-post se corresponde con los mercados de competencia; en el caso del efecto multiplicador de la inversión, hay que dividir los recursos de las empresas en factores de flujo y factores de reserva. Si la demanda se expande, el efecto multiplicador puede reducir o tardar en hacer su efecto si las empresas tienen muchas reservas de mercancías en inventarios, con las cuales se hace frente a la expansión de la demanda; lo mismo pasa cuando Hicks investiga las repercusiones de los costo salariales en las variaciones de los precios. Para hacerlo, divide el mercado laboral en dos sectores: uno, en el que se tiene una relación laboral estable de largo plazo y está regida por un acuerdo o convenio mutuo entre los trabajadores y los contratantes y otro, donde la relación de laboral es efímera y no hay seguridad en el trabajo. Según sea el tipo de trabajo contratado, sus costos repercutirán de manera distinta en los precios, pues éstos se rigen por el costo normal y no por el costo del momento.

Siguiendo este método de Hicks para aplicarlo al mercado mundial, se puede dividir éste en dos partes: la primera es donde existen precios de mercado. Es un mercado abierto a los participantes y las transacciones se hacen entre dos propietarios diferentes. Normalmente este tipo de mercado es lo que se contabiliza como compra y venta de un país con otro país o de una empresa con otra. La segunda parte del mercado mundial, está compuesto por el conjunto de flujos de mercancías que no pasan de un propietario a otro aunque sí fluyen entre los países. Son los bienes y servicios que se trasladan de un país a otro, pero cuyo transporte corre a cargo de la misma empresa: es el traslado de mercancías de una filial a otra o de una filial a la matriz dentro de una misma 
empresa: se conoce como comercio intrafirma. Este tipo de comercio no es un intercambio entre dos propietarios diferentes, sino que el mismo propietario, la misma firma transporta entre sus instalaciones, que están situadas en distintos países, los bienes semiterminados o las partes y materiales que sirven para obtener el producto final (en ocasiones el producto final pasa de un país a otro para ser comercializado).

En este tipo de comercio intrafirma, es difícil predecir el precio de las mercancías intercambiadas, pues eso depende de cómo la empresa aprecia el sistema fiscal del país en que está instalada, tanto como los costos de la mano de obra y de otros insumos, así como los costos de transporte y comunicación de una instalación a otra. En este tipo de comercio, es muy común encontrarse con el fenómeno de la sobre y sub facturación, que las empresas practican para evadir los impuestos locales. Por ello, los precios que se declaran en las facturas de importación y exportación, no necesariamente coinciden con los precios del mercado abierto. Y por tanto el valor de las exportaciones y de las importaciones registradas en un país, puede estar sub o sobre valuado.

Estos dos sectores del mercado mundial, el mercado interfirma y el mercado intrafirma permiten dividir los montos del comercio internacional. Por un lado el comercio tradicional que se efectúa entre las empresas y por el otro, el que está encerrado en la misma firma con diferentes instalaciones en diferentes países. Si el comercio internacional se separa de esa manera y se mide el peso relativo de cada uno, los gobiernos de cada país podrán aplicar políticas de fomento del comercio exterior más idóneas y apropiadas para cada tipo de comercio.

\section{Bibliografía}

Pizano Salazar, Diego, Algunos creadores del pensamiento económico contemporáneo, México, Fondo de Cultura Económica, 1980.

Hicks, J.R., Valor y Capital. México, Fondo de Cultura Económica, 1976 a. Hicks, John, La crisis de la economía keynesiana, Barcelona, Editorial Labor, 1976 b. Hicks, John, Dinero, interés y salarios, México, Fondo de Cultura Económica, 1989 (1982). 